DOI: 10.12731/2658-4034-2020-2-22-31

\title{
FACE-TO-FACE COMMUNICATION, NON-VERBAL BODY LANGUAGE AND PHUBBING: THE INTRUSION IN THE PROCESS
}

\author{
Thseen Nazir \\ Counseling and Guidance Department, Ibn Haldun University, \\ Istanbul, Turkey
}

During evolution, humans evolved with many potential skills, which made them unique than other living beings on the planet. One of the biggest achievements during this evolutionary process was the development of language and the capacity of communication. With the capacity of language, humans are able to share their thought with each other, following a proper medium, and channel in order to do so. This face-to-face communication between two or more individuals follows a specific medium and involves a specific structure, which influences the quality and understanding of a message. In the modern world, we have enhanced all aspects of the communication channel in order to decrease any mental distance among people. Technology has brought about many gadgets into existence, which are very effective in decreasing the physical distance in communication. These gadgets turned out to be very effective and served their purpose well. However, they started having a profound impact on our overall quality of communication and in turn affected our relations, as well as our selves. Nowadays one of the strongest intruding gadgets in our day-to-day life is the smartphone. The use of smartphone during social gatherings, especially during one-to-one communication, has completely changed the way we view our social interactions. This current paper will try to understand face-to-face communication within the context of Phubbing and examine the profound impact of Phubbing on transmission and receiving of messages.

Keywords: Face-to-Face communication; Phubbing; Non-Verbal Communication. 


\section{Introduction}

'Human beings have evolved from apes' is perhaps one of the most important concepts we have all come across in our biological text books from childhood classes. According to the theories of evolution, humans have evolved through millions of years and during this process, we have developed many unique features and characteristics that make us completely different from other animal species. One important and possibly game changing feature is the well-developed communication capabilities that no other species had developed at such a full-fledged capacity. This unique feature makes human beings special. One of the evolutionists, namely Philip Lieberman, emphasizes in his book 'Eve spoke' that amongst the entire species of animals it is only the Homo sapiens who are able to talk and this is the ability that sets us apart from other animals, and it is also a unique gift we have inherited [1]. No doubt, there are many other species that has the ability to communicate but there is a huge difference between the barking dog, the hoot of the owl and the Human speech. This capacity of sharing ideas, thoughts, and information keeps us apart from the rest of all living creatures on this earth. The process of communication is a kind of transaction between two human beings; wherein a person invents and assigns meanings in order to attain certain objectives. Literature regarding communication has put forward many theories related to human communication. Each one of these theories is using a specific approach to understand the process of communication from their own point of view. This paper is not concerned about those issues but rather, the focal point of this paper is to understand the elements of communication and how new human made barriers are influencing it. Therefore, initially we will try to explain what the key elements of human communications are and then we will discuss the barriers influencing it.

\section{Communication and its elements}

Communication is a process, which refers to exchange of ideas, thoughts, and information between two individuals. For successful communication between two individuals, there must be a transaction of information and understandability. The communication process includes 
a Sender, a Receiver, a Medium, and a Message. The process starts with the sender who wants to share his thoughts, ideas or information and is the source in a communication process. The receiver is the person who receives the information, for whom the information was sent by the source. The receiver is also known as the interpreter. The medium is the channel through which the message or information is sent from the sender to the receiver. Lastly, the message is the content or the information that a sender want to relay onto the receiver. These are the basic elements involved in most communication processes. The communication process begins with the message which is sent by the sender, encoded and transmitted through various channels or mediums to the receiver who decodes it or interprets it in a meaningful way [2]. As mentioned above, the process of communication may look simple but there are different factors involved that are responsible for the effectiveness and completion of the communication process. The communication process is dynamic, changing and reciprocal. After analysis of various definitions of communication, we found that each of these definitions emphasize on common aspects of a communication process, which is characterized as the Sender, Medium, Message and Receiver. Beside these elements, communication is comprised of two parts; verbal and nonverbal communication. Verbal part of communication refers to the "words" and the nonverbal communication refers to unspoken words, which comprises gestures, tone of voice, expressions, and body movements etc. The process of communication is not as simple as it looks. In addition to the basic elements in the process, there are many other components involved as well, which are impalpable to the participants and overall, has a very strong effect on the process of communication. One of the impalpable variables, which can influence the entirety of the communication process and its purpose, is the nonverbal aspect of communication.

\section{Nonverbal Communication}

Human communication comprises of two parts i.e. Verbal and Nonverbal communication. Nonverbal communication is made up of those unspoken words, which include facial expressions, gestures, eye contact, 
body posture, tone of voice, hand movements etc. Such expressions are also called silent or sign language. In general, Nonverbal communication includes all communicative behaviors except words. Most of us, without being aware of it, use, perceive and interpret such kind of body language while communicating with others. These nonverbal patterns with the verbal communication patterns are an integral part of interpersonal communication and complete the entire process of transfer of information. Combination of both verbal and nonverbal communication can help convey the messages in a more meaningful and satisfactory way. Nonverbal communication colors the verbal message and supports it in a more significant way [3]. Considering the totality of the communication which consists of the verbal and nonverbal communication, two-third to three-fourth of the communication is made up of the nonverbal part [4].

The facial expressions and body movements play an important role in communicating using the verbal aspects of communication. According to Ekman these facial expressions and body movements can be divided into five categories [5]. These are as follows:

1. Symbolic Movement: - These include facial expressions which are used as a substitute to the verbal language during communication and these substitutes vary according to the culture.

2. Illustrative Movement: - Nonverbal facial expressions are used along with the verbal part of communication in order to clarify the message.

3. Emotion revealing movement: - In this case, nonverbal facial expressions and movements are associated with the mood and emotion of the person.

4. Modulation movement:- Such nonverbal expressions are used to facilitate face-to-face communication; for example, the head and eye movements when a person talks falls into this category.

5. Adaptable movement: - These kinds of nonverbal movements convey certain specific messages such as, greeting via shaking of the hands etc.

Therefore, from the discussion above, we have understood that there are different types of expressions and movements, which we embed to- 
gether with our verbal messages in order to convey messages more clearly and more accurately. These types of expressions along with the verbal content give both the sender and the receiver certain amount of satisfaction and makes communication clearer. Moreover, nonverbal elements of communication along with verbal communication helps individuals to express their ideas and feelings [6]. Thus, it is clear that in order to make the communication process successful, both verbal and nonverbal aspects play an important role. If both the verbal and nonverbal aspects are not communicated or understood, it can lead to misunderstandings or an incompletion of communication. Both of them must be must be clearly channeled and if any one of them is not channeled or embedded, it may influence not only the process but may influence the relationship between the people and outcome of the communication. In recent times, when technology overtook communication channels, there has been a huge shift in the way we socialize. Most of us will agree that these days, we undoubtedly communicate with each other within seconds but these messages communicated through any mode are just hollow words. We do definitely communicate but whether our messages are embedded with emotions and a sense of bonding is debatable. Face-to-face communication these days encounters many hurdles and that too in different forms. One such hurdle is Phubbing, which is influencing our social relations and their outcomes completely.

\section{Phubbing}

In the modern world, smartphones are equipped with many gadgets and its use and compatibility is making it the most usable device ever used by humans. In the current world, it is hard to think of life without such a device. From using it as a device to using it as a phone and allowing us to accesses all the information at the very tips of our fingers, it has changed every aspect of our lives. The importance of this device is unquestionable but the negative impact that this device brings in our lives are yet to be explored to its full extent. One such phenomenon that is associated with this device in the context of social interaction is Phubbing. The term Phubbing is almost a decade old and comes into light after the invention and spread of the excessive use of smart phones. The 
word "Phubbing" actually means Phone snubbing and it came into existence after the merging of these two words. As Nazir states, "Phubbing refers to the intentional or unintentional action of ignoring others during social interactions or events by using smart phones, whether it is checking Facebook, using WhatsApp or other chatting applications or surfing the internet." [7].

The Phubbing phenomenon is very common around us, from family dinners to a couple's bedroom, from class lectures to official meetings etc.; we can observe such behavior in every social gathering. It sometimes seems as if it has become a part of our lives and people have accepted it as a new social norm. During the most recent social activities, $90 \%$ of the participants in the study reported the use of smart phones and around $86 \%$ of others did the same while involved in their social interactions [8]. The intrusion of smart phones into our social lives seems like an inescapable and inevitable change. But the bigger question is how this phenomenon is affecting us and how it can affect our social relations. The current paper will analyze Phubbing phenomenon in the light of communication processing theories and from the point of view of elements of communication. The aim of this paper is to understand the communication process and its elements, how and at what point exactly, the phubbing phenomenon intrudes this communication process and affects it.

The phenomenon of Phubbing is very common nowadays in any social setting involving at least two people. In such a dyad or triad, while a person is trying to communicate or share his feelings, the receiver of the message engages in Phubbing. In such a scenario, according to the basic criteria of successful communication, the receiver may only receive the verbal part of communication and will completely miss the nonverbal aspects of communication. In order to receive the complete message, which includes the verbal as well as the nonverbal parts of communication, the listener or receiver must pay his full attention to the sender and focus his visual senses towards the receiver in order to receive most parts of the message. However, if a sender counters the phubber then most parts of the nonverbal communication will be lost and it will have an impact on 
the complete transmission. Although the Phubber is present physically while not paying attention to the conversation, is not present mentally or emotionally, this kind of a scenario is called "absent-presence" [9]. In addition to this, the person who faces Phubbing may even feel ignored by the phubber during social conversations [10]. In this way, it has a huge impact on social conversations as well as our social relationships. Such behavior also reduces the quality of social relationships as it affects belongingness, self-esteem, sense of meaning and sense of control in life, which are fundamental requirements in life [11]. Phubbing phenomenon is a common behavior nowadays in our society and it is changing the social conversations once and for all. The intrusion is so deep that it is hampering our immediate relationships. This phenomenon is studied more among couples and has been termed as "Partner Phubbing". The people who face Partner Phubbing have reported to feeling lonelier, more Isolated and depressed, and it is becoming one of the root causes of conflicts in relationships [12].

\section{Conclusion and Discussion}

Face is the index of our mind and our messages are made not only of words which we utter verbally, but our communication is also made up of elements beyond those words. Here I want to quote Sigmund Freud as he rightly said:

"He that has eyes to see and ears to hear may convince himself that no mortal can keep a secret. If his lips are silent, he chatters with his fingertips; betrayal oozes out of him at every pore."

Sigmund Freud, Dora: An Analysis of a Case of Hysteria [13].

The aim to quote Sigmund Freud here is to understand the importance of our nonverbal communication. These nonverbal cues and messages make us understand the message, interpret it, and understand it in a proper way. Now the question to be asked is whether, when we receive half the message and don't pay attention to the other half, we will still understand the message and interpret it in the same way as it was intended to be. For complete communication to happen we must attend to not only the verbal part of communication but the nonverbal must be received and interpreted 
properly as well. However, the above-mentioned Phubbing phenomenon is hindering the perception of the second important part of communication i.e. nonverbal messages. For example, when a person is sharing his feelings or emotions with someone who is Phubbing at the same time, it is doubtable whether in these situations actual communication is happening or not. A study conducted with students who faced Phubbing during face-to-face conversations found that $86.2 \%$ felt that the phubber is not listening to them and as a result of which they felt annoyed [14].

Similarly during family dinners, partners sitting side by side on a bed, or a teacher giving lecture, Phubbing can be encountered and in such situations it is important to identify whether the phubber is able to perceive the important parts of nonverbal communication or not and how much it can impact relationships. It has been found that when a person interacts face-to-face and is being Phubbed at the same time, the quality of their interaction goes down [15] and it causes relationships between people to weaken [16]. Some researchers found that when a person faces Phubbing then the interpersonal conversation becomes less meaningful, less personal and it becomes less satisfactory [17].

Our communication comprises of only $7 \%$ of what a listener perceives and the remaining $93 \%$ of what a listener comprehends in communication are the unspoken words, which we call body language and other nonverbal cues [18]. Elaborating on Mehrabian's [19] understanding of communication, as he explained that communication consists of $7 \%$ meanings in the form of words, which a person speaks, and $38 \%$ of communication is a meaning of paralinguistic and 55\% are the facial expressions. So, one can imagine any kind of communication that happens between two people face-to-face under Phubbing circumstances where a phubber is unable to receive complete messages as he is unable to perceive the facial expressions that are embedded into the message. According to the above-mentioned fact about communication a person will be able to perceive only $7 \%$ of communication contents and missing out all other embedded contents. The phubbing phenomenon not only produce the communication barriers but it also develops a social void among people. Among romantic partners it has been found that if encountered phubbing, a partner is sending sub- 
liminal signals to other partner regarding is priorities [20]. Whether it's a working relationship, academic relationships, or an intimate relationship between romantic partners, there is a strong intrusion in our social relations and impact of Phubbing cannot be ignored [21]. This unexpected intrusion in our conversations may create a void and much more unexpected misunderstandings in our communication and most of us are yet not aware of such intrusion in our communication processes and there is much more need to study this unexpected barrier.

\section{References}

1. Lieberman P., Eve Spoke: Human Language and Human Evolution, W.W. Norton, New York, p. 5, 1998.

2. Watson J., Hill A., "A dictionary of communication and media studies", 4th Edition, Arnold, London, 1997, p 251.

3. Novinger T. (2001), Intercultural Communication. A practical guide. University of Texas Press, USA.

4. Novinger T. (2001), Intercultural Communication. A practical guide. University of Texas Press, USA.

5. Ekman P. (2007), Emotions Revealed, Recognizing Faces and Feelings to Improve Communication and Emotional Life, Times Books, Henry Holt and Company, New York.

6. Sharifiabad M. and Vali S., 2011, A Comparative Study of Native and Non-Native Body Language. Journal of Intercultural Communication, 26:6.

7. Nazir T. \& Pişkin M. (2016) Pubbing: A Technological Invasion, Which Connected the Word But Disconnected Humans. The International Journal of Indian Psychology, 3, 39-46.

8. Ranie L., \& Zickuhr K. (2015). Americans' views on mobile etiquette. Pew Research Center.

9. Katz E. and Aakhus M. (2002) Perpetual Contact: Mobile Communication, Private Talk, Public Performance. Cambridge University Press, Cambridge, UK.

10. Nazir T. (2017). Attitude and Emotional Response among University Students of Ankara towards Phubbing. International Journal of Multidisciplinary Educational Research, 6, 5 . 
11. Gerber J. and Wheeler L. (2009) On Being Rejected a Meta-Analysis of Experimental Research on Rejection. Perspectives on Psychological Science, 4, 468-488.

12. Krasnova H., Abramova O., Notter I. and Baumann A. (2016) Why Pubbing Is Toxic for Your Relationship: Understanding the Role of Smartphone Jealousy among "Generation Y" Users. Research Papers. 109.

13. Mieke. B. (2004). Narrative Theory: Special topics. Routleg Publishing House.

14. Nazir T. \& Pişkin M. (2016) Pubbing: A Technological Invasion, Which Connected the Word But Disconnected Humans. The International Journal of Indian Psychology, 3, 39-46.

15. Abeele M.M.V., Antheunis M.L., \& Schouten A.P. (2016). The effect of mobile messaging during a conversation on impression formation and interaction quality. Computers in Human Behavior, 62, 562-569.

16. Nazir T. \& Pişkin M. (2016) Pubbing: A Technological Invasion, Which Connected the Word But Disconnected Humans. The International Journal of Indian Psychology, 3, 39-46.

17. Seppälä E. (2016) The Happiness Track: How to Apply the Science of Happiness to Accelerate Your Success. Piatkus Books, London.

18. Mehrabian A. (1970). Tactics of Social Influence. Prentice-Hall, Englewood Cliffs, New Jersey.

19. Mehrabian A. (1970). Tactics of Social Influence. Prentice-Hall, Englewood Cliffs, New Jersey.

20. McDaniel B.T. \& Coyne S.M. (2014) Technoference: the interference of technology in couple relationships and implications for women's personal and relational well-being. Psychology of Popular Media Culture.

21. Nazir T. \& Bulut S (2019). Phubbing: a phenomenon that is mending social relationships. Siberian Journal of Psychology. № 74, pp. 101-109. 